\title{
Um Método Heurístico para Planejamento de Redes de Troncos Digitais em Áreas Urbanas: Utilização de Fibras Ópticas
}

\author{
Anilton Salles Garcla e Hermano de M. F. Tavares
}

No atendimento da demanda por serviços urbanos de telecomunicaçōes, existem diferentes alternativas quanto às facilidades de transmissão a serem instaladas em cada arco da rede. Tais alternativas podem apresentar custos e capacidades de circuitos significativamente diferentes. Obter uma solução viável de custo mínimo é um problema de otimização, de natureza combinatória, complicado pela existência de custos não lineares e um grande número de variáveis. Neste trabalho \& apresentado um método heurístico que permite determinar uma boa solução para a rede de troncos digitais, onde há a competição, em custo, entre os sistemas PCM de 30 canais e fibras ópticas de 480 canais. São também discutidos os principais resultados obtidos em uma aplicação para a rede digital de São Paulo.

\section{Introdução}

A expansão da demanda por serviços telefônicos em áreas locais será, em geral, atendida utilizando-se tecnologia digital. Este novo ambiente tecnológico requer o desenvolvimento de metodologias computacionais para planejamento da rede de troncos em função, por exemplo, da utilização de fibras ópticas (480 canais, $34 \mathrm{Mbit} / \mathrm{s}$ ) no entroncamento digital.

O tratamento conjunto dos entrocamentos em PCM ("Pulse Code Modulation") de 30 canais e sistemas ópticos de 480 canais apresenta um elevado grau de complexidade devido às não linearidades nos custos de entroncamento provocadas pela modularidade dos equipamentos.

Neste trabalho é apresentado um método heurístico para planejamento da rede de troncos digitais, originalmente desenvolvido por Garcia [1] ; ênfase particular é dada ao dimensionamento de fibras ópticas com transmissão a $34 \mathrm{Mbit} / \mathrm{s}$.

Inicialmente, é dada uma descrição do problema e de como ele se insere na metodologia de planejamento da rede de troncos. Em seguida, são discutidos os principais aspectos do modelo matemático. É feita uma rápida caracte-

A.S. Garcia é Engenheiro da Elebra Telecon Ltda, Rua Alfredo da Costa Figo 255, 13100 Campinas SP e Professor Adjunto do Centro Tecnológico da Universidade Federal do Esplrito Santo.

H.M.F. Tavares é Professor da Faculdade de Engenharia Elétrica da UNICAMP, Campinas SP. 
rização computacional do programa, com uma aplicação para a rede de troncos digitais da cidade de São Paulo. Finalmente, são apresentadas as principais conclusões sobre o sistema desenvolvido, suas extensões e sugestões para continuidade do trabalho.

\section{Descrição do Problema}

Um estudo de planejamento de redes telefônicas locais em áreas multicentrais compreende, em linhas gerais, dois problemas: Estudo do Plano Fundamental e Cálculo/Otimização das Interligações. No primeiro problema procura-se obter o número e localização dos novos centros de fios, as ampliações sugeridas para os centros de fios existentes, um cronograma físico de instalação para asimplantações/ampliações e a afiliação dos assinantes às centrais nos vários períodos de planejamento. No segundo, a preocupação básica é com a otimização da rede de troncos que compreende o dimensionamento de circuitos telefônicos para atender a demanda de comunicações entre as centrais e a forma pela qual estes circuitos são roteados através da rede física.

Neste trabalho, é proposta uma abordagem para o planejamento da rede de troncos, levando-se em conta a digitalização dos equipamentos de transmissão de modo a considerar os entroncamentos em 30 canais (2 Mbit/s) e 480 canais ( $34 \mathrm{Mbit} / \mathrm{s})$.

O planejamento da rede de troncos consiste, fundamentalmente, em otimizar a interligação entre as centrais telefônicas, respeitando os níveis de qualidade de serviço estabelecidos para as chamadas e as limitações tecnológicas dos equipamentos de comutação e transmissão. A abordagem tradicional deste estudo é feita em duas etapas: Cálculo do Entrocamento e Otimização do Roteamento.

Inicialmente, houve uma preocupação maior com o problema de Cálculo do Entroncamento (determinação da quantidade de circuitos telefônicos entre as centrais), destacando-se os estudos desenvolvidos por Rapp [2] e [3], Wilkinson [4] e Wällstrom [5]. Tal esforço se justifica porque os equipamentos de comutação analógica apresentam regras rígidas para encaminhamento das chamadas, acessibilidade limitada aos troncos de saida e custos elevados do entroncamento. Os circuitos telefônicos eram, em geral, uniroteados. Observa-se, nesta direção, um grande esforço brasileiro, ligado à empresas do sistema TELEBRAS, no desenvolvimento de ferramentas computacionais para planejamento da rede de troncos, destacando-se, dentre outros, os trabalhos de Moreno [6], Reis [7], Tiba, Solon e Uehara [8] e Shibata $[9]$.

A crescente utilização, a partir de meados da década de 70 , da tecnologia de comutação digital provocou, como decorrência, uma digitalização cada vez maior das interligações entre as centrais. Há, portanto, um período em que as duas tecnologias (analógica e digital) são utilizadas simultaneamente.

42 
Robin e Treves [1(1] propuseram que o estudo de redes mistas fosse feito separando-as em planos, em função da tecnologia empregada. Cada plano seria abordado separadamente.

No estudo do entroncamento digital, apesar dos equipamentos de comutação apresentarem acessibilidade plena aos troncos de saída e regras de encaminhamento mais flexiveis, a necessidade de interfaces padronizadas e a modularidade dos sistemas digitais provocam uma não linearidade nos custos de entroncamento e uma maior utilização dos sistemas de transmissão digitais de alta capacidade. Aparece, então, a necessidade de considerar de modo mais rigoroso alguns aspectos do problema de roteamento, tais como: formulação matemática do problema que considere a utilização de vários meios de transmissão, McCallum [11] ; um tratamento da segurança da transmissão, por exemplo, através do multiroteamento das demandas, Minoux [12], Garcia, Tavares, Ribeiro e Bim [13] ; um estudo do roteamento em horizontes de longo prazo comtemplando aspectos de evolução tecnológica, Yaged [14], Garcia, Tavares, Ribeiro e Guerra [15] ; a consideração de várias hierarquias para a transmissão digital.

Este trabalho tem a preocupação voltada para o plano digital, permitindo tratar os aspectos de segurança da transmissão e a otimização do roteamento, considerando os entroncamentos em $2 \mathrm{Mbit} / \mathrm{s}$ (PCM de 30 canais) e 34 Mbit/s (fibras ópticas de 480 canais). Na solução destes problemas, são utilizados algoritmos de fluxos em redes combinados com técnicas de programação matemática.

O problema de planejamento da rede de troncos digitais deve ser considerado num intervalo plurianual, chamado horizonte de planejamento, onde a demanda a ser atendida é um parâmetro temporal. Em geral, o horizonte é dividido em períodos e estudos tipo "cross-section" efetuados para os anos finais de cada um dos períodos. É necessário coordenar os resultados dos diversos períodos de forma a obter boas soluções em termos de investimento global sobre todo o horizonte de planejamento. Deve-se ressaltar que uma eventual ociosidade de canais num período pode ser desejável para fazer face ao crescimento da demanda futura; fatos como este, de natureza essencialmente dinâmica, só podem ser captados numa abordagem que considere diversos períodos (multiperíodos). A abordagem apresentada neste artigo é estática, do tipo "cross-section" (uniperíodo). Em desenvolvimento futuro tentaremos abordar o problema multiperíodo.

\section{O Modelo Matemático}

O propósito inicial do planejamento da rede de troncos é atender de modo otimizado a necessidade de circuitos telefônicos ențre as centrais (matriz de troncos), obtida levando-se em conta o interesse de tráfego entre as centrais, o número de assinantes de cada central, as regras de encaminhamento e o grau de serviço definidos para as ligações. 
A rede de troncos é descrita por meio de uma estrutura de nós (centros de fios e pontos de passagem) e arcos (galerias de dutos) contendo informações sobre o comprimento e a disponibilidade de meios de transmissão em cada arco. A consideraçāo dos entroncamentos em $2 \mathrm{Mbit} / \mathrm{s}$ e $34 \mathrm{Mbit} / \mathrm{s}$ é facilitada pela construção dos planos de transmissão em PCM de $2 \mathrm{Mbit} / \mathrm{s}$ (PTP) e em fibras ópticas de $34 \mathrm{Mbit} / \mathrm{s}$ (PTF). Para a definição das rotas (associação de um caminho físico com um meio de transmissão) há a necessidade de determinar os caminhos físicos entre as centrais em cada um dos planos. A Fig. 1 ilustra como podem ser visualizadas as rotas nesses planos. Nesta figura, I e J representam centrais de origem e destino de tráfego, respectivamente, enquanto $P_{k}$ e $P_{\text {I }}$ são pontos de mudança de hierarquia de transmissão, cada um deles constituindo o que se denomina uma porta.

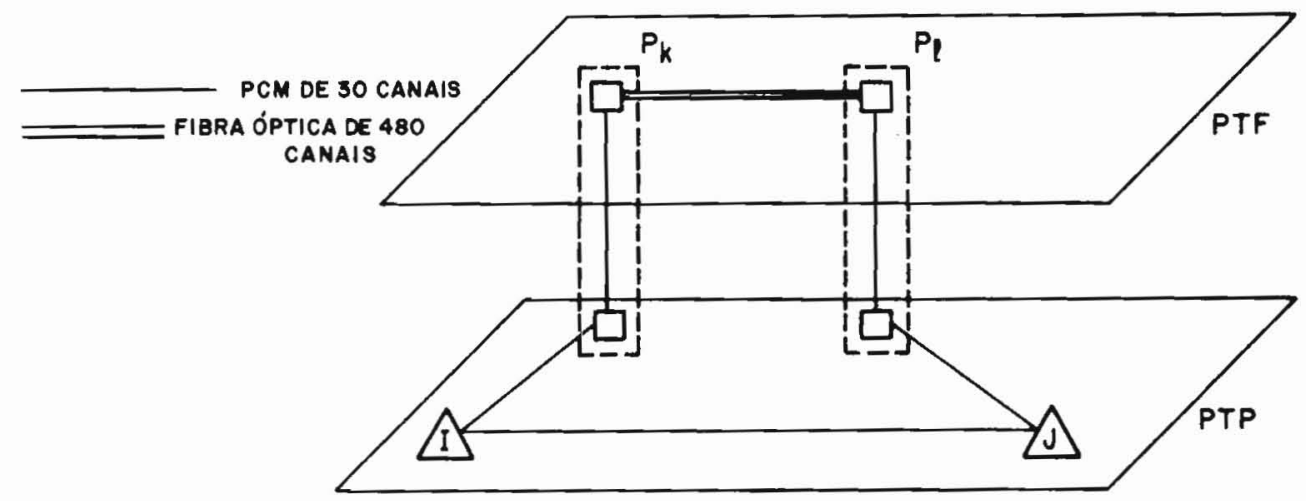

Figura 1. Esquema de transmissão nos planos digitais.

A determinação das rotas no plano PTP é feita utilizando-se algoritmos de fluxos em redes para obter: os $k$ menores caminhos entre cada par de estações; os dois caminhos disjuntos de soma mínima entre cada par de estações. As rotas no plano PTF são obtidas combinando-se algoritmos de fluxos em redes com técnicas de programação dinâmica; são determinados os três melhores caminhos entre duas estações passando por duas portas.

Dois tipos de restrições são considerados no problema: disponibilidade dos meios de transmissão existentes nos arcos; atendimento da demanda de troncos entre as centrais. A consideração das disponibilidades é importante para possibilitar a aplicação do modelo tanto em redes novas como onde já existam facilidades de transmissão instaladas. Este primeiro tipo de restrição é tratado utilizando-se uma formulação arco-rota (arco-caminho) para descrição das rotas (matriz arco-rota), onde cada linha da matriz corresponde à utilização de um meio de transmissão em uma galeria de dutos. $O$ tratamento do segundo tipo de restrição é obtido, adotando-se uma caracterização em que a demanda de troncos entre duas centrais é o produto e o número 
de circuitos escoados por uma determinada rota é o fluxo. Representando por $X_{r}$ o fluxo na rota $r$ por $d^{p}$ a demanda do produto $p$ e por $R(p)$ o conjunto de rotas associadas ao produto $\mathrm{p}$, tem-se

$$
\sum_{r \in R(p)} X_{r}=d^{p}
$$

É fácil notar que o atendimento das demandas dos vários produtos pode provocar a necessidade de compra de equipamentos de transmissão nos arcos da rede. Definem-se então um vetor de fluxos $X=\left\{X_{r}\right\}$, um vetor de disponibilidades $S=\left\{S_{a}\right\}$ e um vetor de compras $Y=\left\{Y_{a}\right\}$, onde $S_{a}$ e $Y_{a}$ representam, respectivamente, os números de circuitos disponíveis e a serem adquiridos no arco a. Define-se também uma matriz arco-rota $\mathbf{A}$ $=\left\{A_{i j}\right\}$, onde

$$
A_{i j}=\left\{\begin{array}{l}
1, \text { se o arco i participa da rota } j \\
0, \text { em caso contrário }
\end{array}\right.
$$

Finalmente, representando por NP o número de produtos, podemos caracterizar as restrições do problema por

$$
\left\{\begin{array}{l}
A X \leqslant S+Y \\
\sum_{r \in R(p)} X_{r}=d !, p=1,2, \ldots, N P \\
X \geqslant 0, Y \geqslant 0
\end{array}\right.
$$

Observe-se que o modelo adotado permite a consideração de um número arbitrário de rotas alternativas, em quaisquer dos planos, entre cada par de centrais. É importante observar também neste modelo que, embora de natureza aleatória, as demandas são consideradas como parâmetros determinísticos, definidos para os anos finais de cada período de planejamento.

Levando-se em conta que um dos propósitos do planejamento é minimizar os investimentos necessários para atendimento da demanda, o modelo indicado anteriormente considera dois tipos de custos: custo dos fluxos nas rotas e custo de compra nos arcos. Para os fluxos nas rotas, supõe-se que o custo de ligar enlaces é proporcional ao número de circuitos na rota. Penalizações convenientes devem ser aplicadas quando os aspectos de segurança são considerados. Com relação às compras, é dado um tratamento diferenciado para os arcos dos planos PTP e PTF.

Para um arco do plano PTP, supõe-se que a função de custo assume a forma mostrada na Fig. 2, onde: $N$ representa 0 número de sistemas PCM 
de $2 \mathrm{Mbit} / \mathrm{s}$ já roteados no arco; $\mathrm{C}$ representa o número de pares disponíveis no arco para sistemas PCM de $2 \mathrm{Mbit} / \mathrm{s}$ : CR designa o custo dos regeneradores no arco por sistema PCM; e CT designa o custo total (cabo + regeneradores) no arco por sistema PCM.

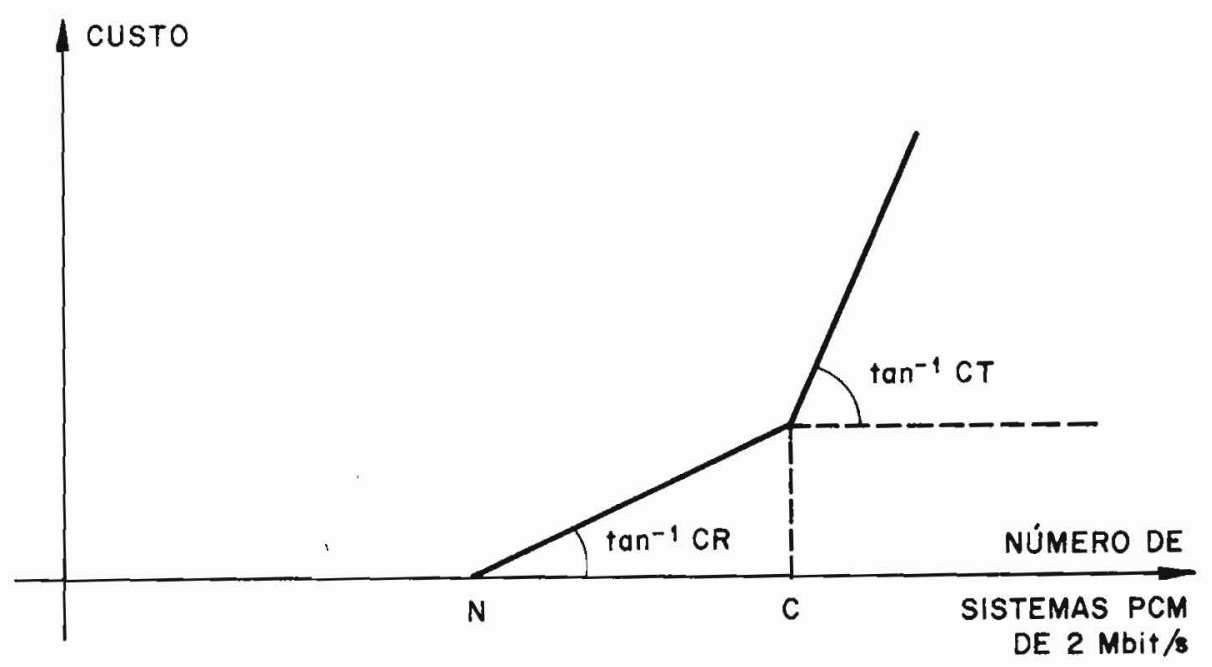

Figura 2. Custo para a compra em um arco do plano PTP.

Para os arcos do plano PTF, é preciso visualizar o esquema de transmissão entre duas portas, que são os pontos onde há mudança na hierarquia de transmissão (2 Mbit/s para $34 \mathrm{Mbit} / \mathrm{s}$ e $34 \mathrm{Mbit} / \mathrm{s}$ para $2 \mathrm{Mbit} / \mathrm{s}$ ), conforme representado na Fig. 3. Nesta figura, a e b representam linhas de transmissão $\mathrm{PCM}$ de $2 \mathrm{Mbit} / \mathrm{s}$ (30 canais) e $8 \mathrm{Mbit} / \mathrm{s}$ (120 canais), respectivamente, enquanto $\mathrm{c}$ representa linhas de transmissão em fibra óptica de $34 \mathrm{Mbit} / \mathrm{s}$ (480 canais). Ainda na Fig. 3, tem-se que MUX2 e MUX3 são equipamentos de multiplexação que agrupam, respectivamente, 4 sistemas PCM de $2 \mathrm{Mbit} / \mathrm{s}$ em 1 sistema PCM de $8 \mathrm{Mbit} / \mathrm{s}$ e 4 sistemas PCM de $8 \mathrm{Mbit} / \mathrm{s}$ em 1 sistema de $34 \mathrm{Mbit} / \mathrm{s}$ (correspondente portanto a 16 sistemas PCM de $2 \mathrm{Mbit} / \mathrm{s}$ ). Assinale-se finalmente que TL3 representa o equipamento de terminação de linha de $34 \mathrm{Mbit} / \mathrm{s}$.

Para os arcos do plano PTF, a curva de custo assume a forma representada na Fig. 4, onde d representa a parte fixa dos custos dos equipamentos de transmissão em $34 \mathrm{Mbit} / \mathrm{s}$ para cada conjunto de até 16 sistemas de $2 \mathrm{Mbit} / \mathrm{s}$ (modularidade) e e representa o custo dos equipamentos de multiplexação dos sistemas de $8 \mathrm{Mbit} / \mathrm{s}$. $O$ custo d inclui dois equipamentos MUX3, tois TL3, os gastos com fibras indispensáveis para a transmissāo no plano गTF, bem como repetidores ópticos na proporção de um repetidor a cada $10 \mathrm{~km}$, em média, para o sistema de $34 \mathrm{Mbit} / \mathrm{s}$. O custo e corresponde a tois equipamentos MUX2. 


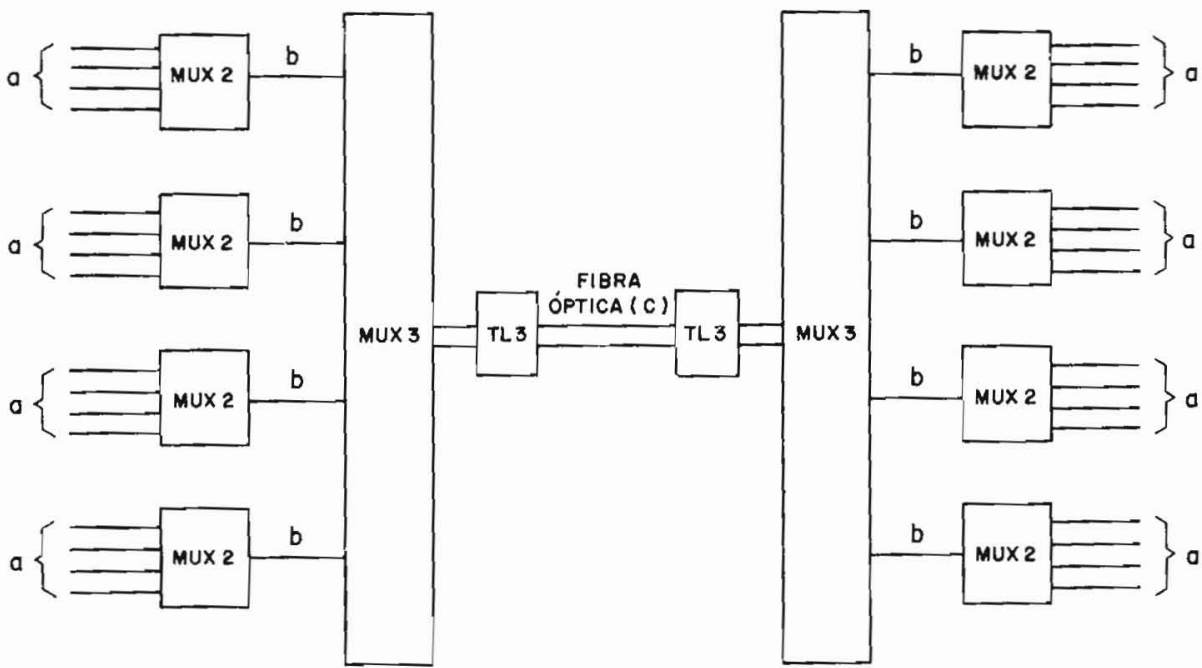

Figura 3. Representação do esquema de transmissão éntre duas portas.

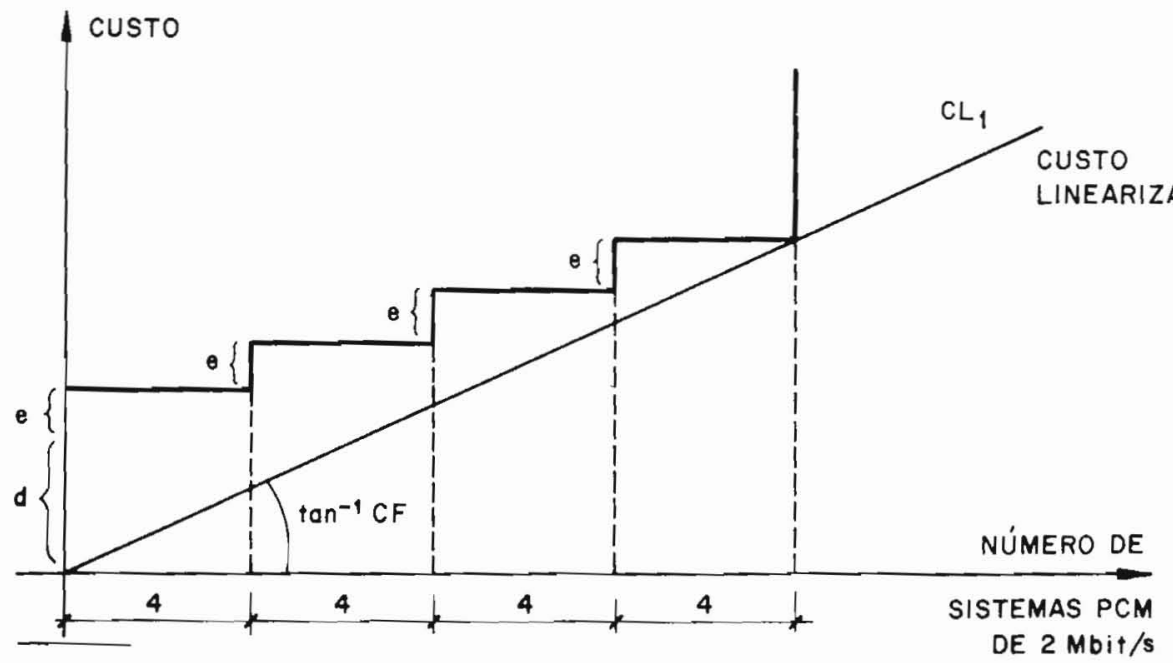

Figura 4. Função de custo para a compra nos arcos do plano PTF.

Problemas envolvendo este tipo de custo, não linear e discreto, são de difícil tratamento matemático. Exigem, para sua solução, a consideração de variáveis inteiras ou o tratamento via procedimentos heurísticos. Neste trabalho foi utilizado um tratamento heurístico que consiste de aproximações lineares sucessivas da função de custo, gerando uma estrutura linear por partes na resolução do problema. 
O problema resultante consiste, portanto, em

$$
\underset{\mathbf{X}, \mathbf{Y}}{\operatorname{Minimizar}} F(X, Y)=\sum_{j=1}^{N R} f_{j}\left(X_{j}\right)+\sum_{a=1}^{M} 8_{a}\left(Y_{a}\right)
$$

atendidas as restrições expressas por (3). Em (4), NR e $M$ representam os números totais de rotas e de arcos, respectivamente, enquanto $f_{j}\left(X_{j}\right)$ é o custo de se ter um fluxo $X_{j}$ na rota $j$ e $\delta_{a}\left(Y_{a}\right)$ é o custo associado à compra de $Y_{a}$ circuitos no arco a. As funções $f_{j}\left(X_{j}\right)$ são, em princípio, lineares e correspondem ao custo de ligar circuitos em cada rota j. Quando são considerados os aspectos de segurança da transmissão elas tornam-se lineares por partes e convexas.

A forma do problema de minimização expresso por (4) e (3) é propícia à utilização do método "Generalized Upper Bounding"(GUB) [11] para programação linear por partes.

O procedimento heurístico utilizado para as compras nos arcos do plano PTF é composto pelos três passos descritos a seguir.

PASSO 1 (P1) - Obtenção de uma solução inicial "otimista" no plano PTF, utilizando uma linearização dos custos com coeficiente

$$
C F=(d+4 e) / 16
$$

conforme indicado na Fig. 4.

PASSO 2 (P2) - Linearização corretiva de passos anteriores, procurando diminuir a diferença entre o custo real $\left(C_{R}\right)$ e o custo linearizado $(C L)$, conforme indicado na Fig. 5.

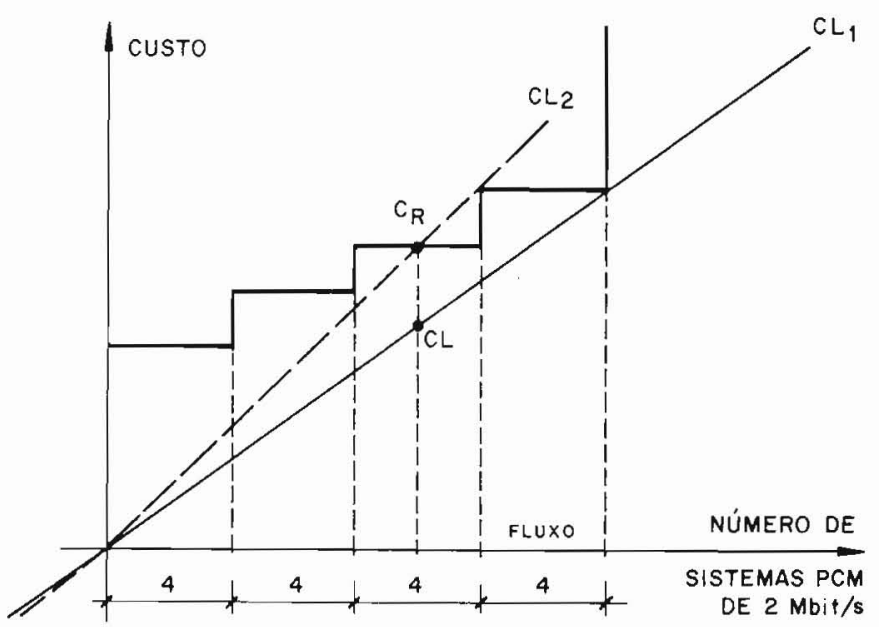

Figura 5. Função de custo $\mathrm{CL}_{2}$ correspondente ao passo 2. 
PASSO 3 (P3) - Aproveitamento, em função da modularidade dos sistemas de $8 \mathrm{Mbit} / \mathrm{s}$, das disponibilidades eventualmente existentes, sem aumento do custo real. Utiliza-se para isto uma linearização por partes e convexa do custo real, conforme mostrado na Fig. 6.

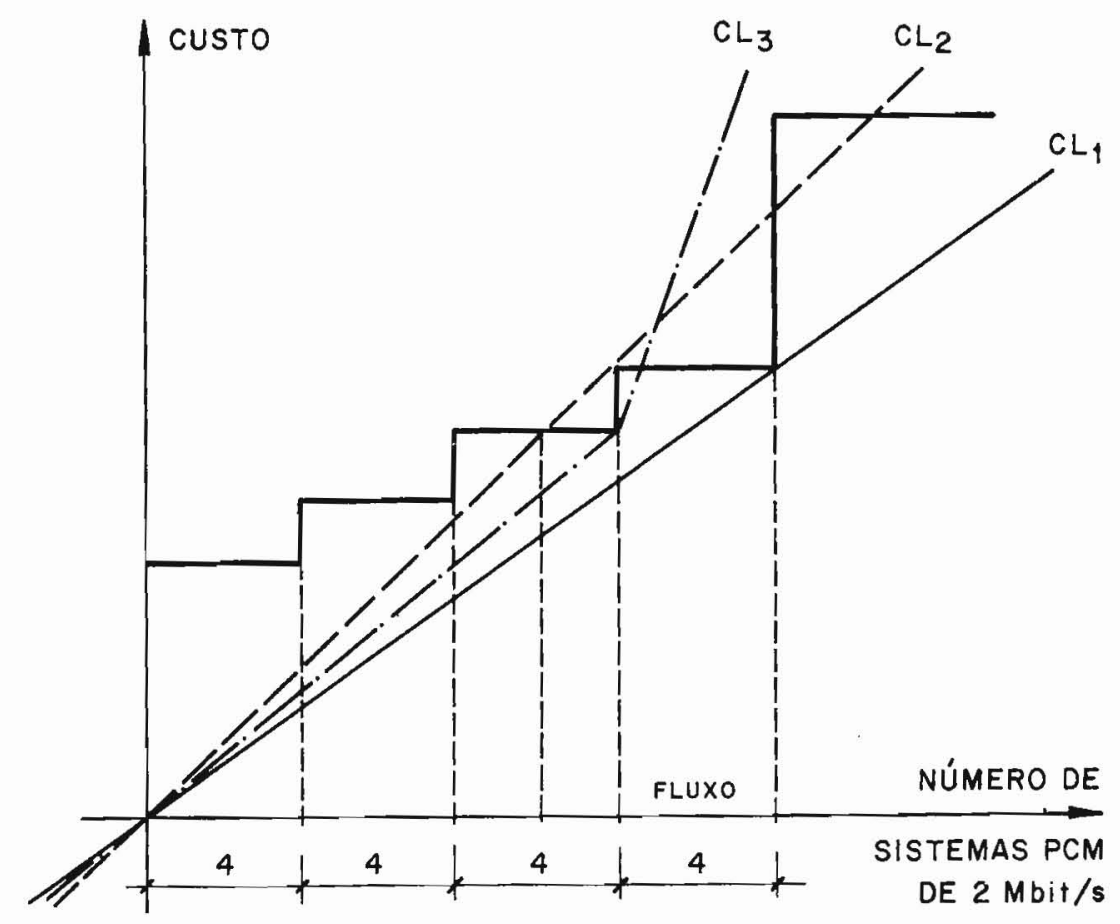

Figura 6. Função de custo $\mathrm{CL}_{3}$ correspondente ao passo 3.

A otimização dos custos é obtida combinando-se, em diferentes seqüências, os passos mencionados acima. O passo 1 é utilizado para inicialização do processo. Em qualquer seqüência utilizada é aconselhável concluir a otimização com a execução do passo 3 ; tenta-se, assim, a plena utilização das disponibilidades já pagas, no plano PTF.

Os modelos anteriores para resolução do problema de roteamento, Reis [7] e Shibata [9], não contemplam a modularidade dos sistemas de transmissão digitais nem a utilização de fibras ópticas de 480 canais. Além disso, tratam do roteamento para a variação da demanda de um período para outro.

Observe-se também que o problema tratado no presente artigo é de natureza combinatória e sua resolução exata demanda a utilização de um grande número de variáveis discretas, gerando um modelo matemático de enorme complexidade e ao qual estão associados tempos computacionais proibitivos. 0 procedimento heurístico apresentado não permite estabelecer, a priori, 
o grau de precisão da solução alcançada em relação à solução exata. Entretanto, os três passos P1, P2, P3 foram idealizados de forma a criar oportunidades que permitam avaliações de limitantes inferiores para a solução ótima. Este fato será ilustrado na Seção 5.

Um procedimento análogo foi recentemente apresentado por Baybars e Edahl [16], onde é considerado um modelo matemático que contempla as mesmas restrições representadas em (3), mas que se diferencia do presente trabalho na representação dos custos nas rotas. Em [16], é adotada uma função 'de custo mais simplificada, onde não é possível a competição entre os vários sistemas de transmissão em uma determinada rota. Entretanto, Baybars e Edhal não se restringem a um tratamento "cross-section" (uniperíodo) e abordam o problema de roteamento para um horizonte de longo prazo (multiperíodo) podendo considerar os aspectos dinâmicos do problema.

\section{Caracterização Computacional}

O diagrama de blocos apresentado na Fig. 7 procura ilustrar, de maneira simplificada, os aspectos envolvidos na modelagem matemática desenvolvida para o problema.

O programa computacional correspondente foi desenvolvido em computador PDP-10, utilizando a linguagem FORTRAN 77 . Versões do programa para computadores das linhas IBM e VAX encontram-se disponíveis e já foram testadas em algumas situações reais. Trata-se de um sistema de médio porte, contendo aproximadamente 5.000 instruções.

\section{Uma Aplicação à Rede de São Paulo}

Com o objetivo de testar o comportamento do programa, o mesmo foi utilizado em várias situações reais. Neste trabalho, é apresentada uma síntese dos resultados obtidos para a rede de troncos digitais da cidade de São Paulo.

A rede original em estudo, mostrada na Fig. 8 , inclui 44 estações telefônicas, 44 nós, 81 arcos e 14 portas. Após a geração das rotas nos planos PTP e PTF é obtida a estruturação dos dados para o processo de otimização. O modelo matemático resultante apresenta as seguintes características: 426 produtos, 2.554 variáveis de fluxo, 181 linhas na matriz arco-rota (A) do sistema de restrições e 1.274 sistemas PCM de $2 \mathrm{Mbit} / \mathrm{s}$ a rotear.

A título de ilustração, devemos assinalar que caso este problema fosse resolvido por um algoritmo exato, haveria a necessidade de se utilizar pelo menos 91 variáveis inteiras correspondentes à decisão instala/não instala sistemas de $34 \mathrm{Mbit} / \mathrm{s}$ entre todos os possíveis pares de portas. Variáveis inteiras adicionais, correspondentes à instalação de equipamentos MUX2, certamente seriam necessárias. Problemas deste porte são de difícil tratamento computacional. 


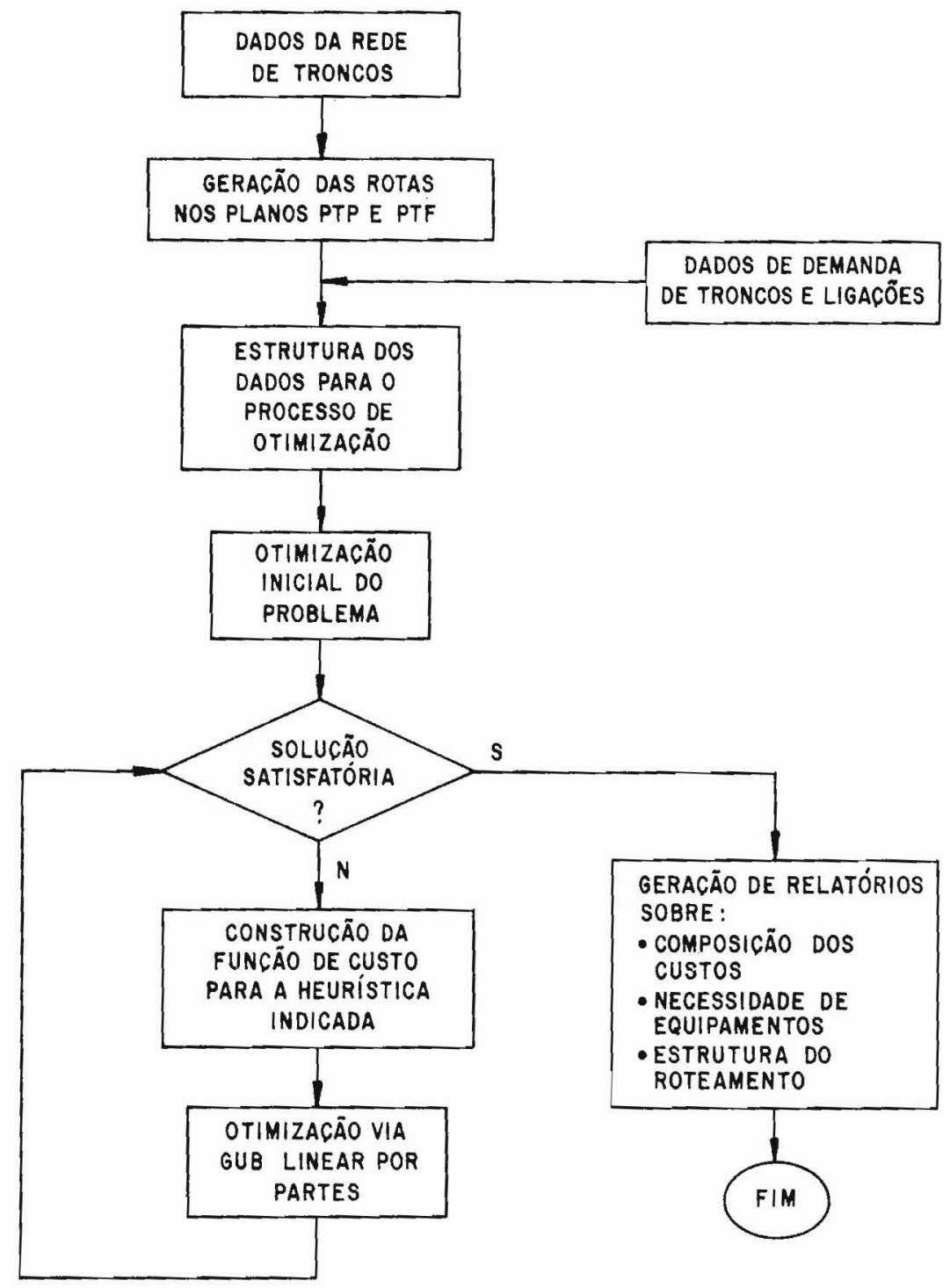

Figura 7. Diagrama de blocos simplificado do programa.

Os principais resultados para duas seqüências diferentes dos passos do procedimento heurístico anteriormente descrito são apresentados. Neles podem ser verificados o carregamento dos arcos no plano PTF, as necessidades de equipamentos e os dados de custo para cada uma das combinações. As seqüências utilizadas foram (P1, P2, P3) e (P1, P3, P2, P3). 


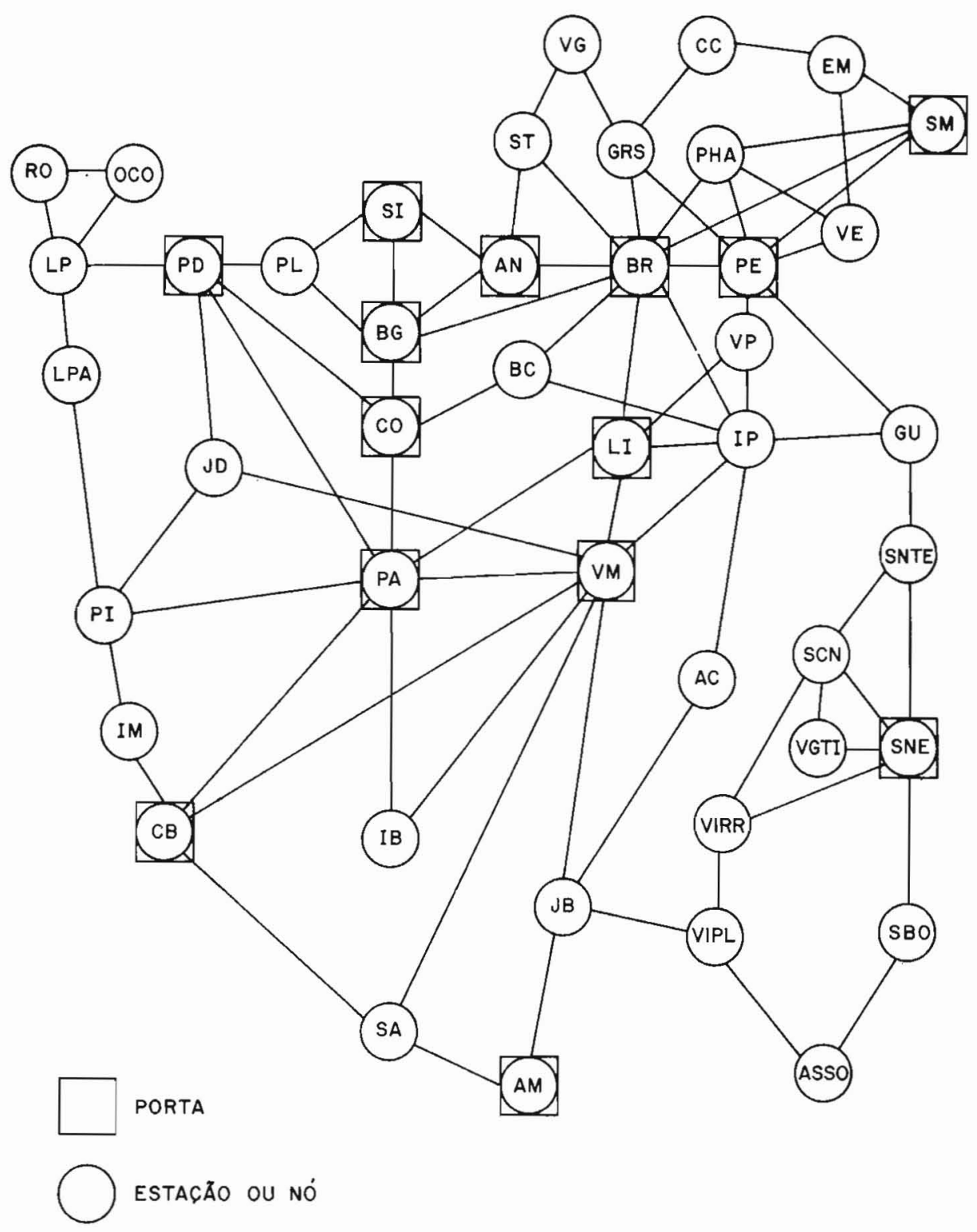

Figura 8. Rede de São Paulo com 14 portas.

5.1. Resultados para a Seqüência (P1, P2, P3)

Na Tabela 1, é mostrado o carregamento dos arcos no plano PTF, após cada passo. A Tabela 2 ilustra os dados de custo e necessidades de equipamento correspondentes. 


\begin{tabular}{|c|c|c|c|c|c|}
\hline & & & \multicolumn{3}{|c|}{ FLUXO NOS ARCOS } \\
\hline \multicolumn{2}{|c|}{ ARCOFIBRA } & \multirow{2}{*}{$\frac{\text { COMPRIMENTO }(\mathrm{m})}{11600}$} & \multirow{2}{*}{$\frac{P 1}{14}$} & \multirow{2}{*}{$\frac{\mathrm{P} 2}{0}$} & \multirow{2}{*}{$\frac{\mathrm{P3}}{0}$} \\
\hline PD & LI & & & & \\
\hline PD & $\mathrm{CB}$ & 17900 & 6 & 0 & 0 \\
\hline PD & $\mathrm{AM}$ & 22100 & 21 & 16 & 16 \\
\hline PD & $\mathrm{PE}$ & 18400 & 10 & 10 & 10 \\
\hline PD & $S M$ & 32300 & 1 & 0 & 0 \\
\hline PD & SNE & 32100 & 10 & 0 & 0 \\
\hline $\mathrm{CO}$ & $\mathrm{CB}$ & 13700 & 6 & 0 & 0 \\
\hline $\mathrm{CO}$ & AM & 17900 & 9 & 0 & 0 \\
\hline $\mathrm{CO}$ & $\mathrm{PE}$ & 12700 & 2 & 0 & 0 \\
\hline $\mathrm{CO}$ & $S M$ & 26600 & 1 & 0 & 0 \\
\hline $\mathrm{CO}$ & SNE & 26800 & 4 & 0 & 0 \\
\hline PA & AM & 14500 & 5 & 0 & 0 \\
\hline PA & PE & 16100 & 3 & 0 & 0 \\
\hline PA & $S M$ & 30000 & 2 & 0 & 0 \\
\hline PA & SNE & 24500 & 12 & 26 & 26 \\
\hline VM & $A M$ & 10800 & 12 & 0 & 0 \\
\hline VM & BR & 9500 & 4 & 0 & 0 \\
\hline VM & $P E$ & 16100 & 2 & 0 & 0 \\
\hline VM & SM & 30700 & 1 & 0 & 0 \\
\hline VM & SNE & 20800 & 4 & 0 & 0 \\
\hline SI & LI & 9900 & 2 & 0 & 0 \\
\hline SI & $P E$ & 14300 & 3 & 0 & 0 \\
\hline SI & $S M$ & 28200 & 1 & 0 & 0 \\
\hline SI & SNE & 29300 & 4 & 0 & 0 \\
\hline$B G$ & $\mathrm{CB}$ & 4500 & 1 & 0 & 0 \\
\hline$B G$ & $A M$ & 18700 & 28 & 28 & 28 \\
\hline$B G$ & PE & 12600 & 3 & 0 & 0 \\
\hline$B G$ & $S M$ & 26500 & 3 & 0 & 0 \\
\hline$B G$ & SNE & 27600 & 8 & 12 & 12 \\
\hline AN & LI & 9700 & 1 & 0 & 0 \\
\hline AN & $\mathrm{CB}$ & 16400 & 2 & 0 & 0 \\
\hline AN & $S M$ & 25800 & 2 & 0 & 0 \\
\hline LI & $\mathrm{CB}$ & 11300 & 16 & 16 & 16 \\
\hline $\mathrm{LI}$ & $A M$ & 15200 & 8 & 0 & 0 \\
\hline $\mathrm{LI}$ & $\mathrm{PE}$ & 12400 & 11 & 0 & 0 \\
\hline LI & SM & 26300 & 3 & 0 & 0 \\
\hline LI & SNE & 23400 & 11 & 11 & 15 \\
\hline $\mathrm{CB}$ & AM & 11500 & 49 & 48 & 48 \\
\hline $\mathrm{CB}$ & BR & 16400 & 2 & 0 & 0 \\
\hline $\mathrm{CB}$ & $\mathrm{PE}$ & 23000 & 4 & 0 & 0 \\
\hline$A M$ & $\mathrm{BR}$ & 20300 & 9 & 0 & 0 \\
\hline $\mathrm{AM}$ & $\mathrm{PE}$ & 26900 & 7 & 0 & 0 \\
\hline$A M$ & $S M$ & 41500 & 1 & 0 & 0 \\
\hline AM & SNE & 23200 & 11 & 9 & 10 \\
\hline$B R$ & $S M$ & 21200 & 1 & 0 & 0 \\
\hline $\mathrm{BR}$ & SNE & 22900 & 3 & 0 & 0 \\
\hline $\mathrm{PE}$ & SM & 16200 & 10 & 0 & 0 \\
\hline PE & SNE & 15600 & 3 & 0 & 0 \\
\hline SM & SNE & 31800 & 1 & 0 & 0 \\
\hline \multicolumn{3}{|c|}{ TOTAL DO SISTEMA } & 337 & 176 & 181 \\
\hline
\end{tabular}

Tabela 1. Carregamento nos arcos do plano PTF para a seqüência (P1, P2, P3). 


\begin{tabular}{l|c|c|c} 
& \multicolumn{3}{|c}{ PASSOS } \\
\cline { 2 - 4 } & P1 & P2 & P3 \\
\hline CUSTO LINEARIZADO (10 3 OTN) & 746,1 & 812,1 & 800,8 \\
CUSTO REAL (10 3 OTN) & 1015,4 & 807,8 & 804,3 \\
CUSTO NO PLANO PTP (10 ${ }^{3}$ OTN) & 532,5 & 678,4 & 674,0 \\
CUSTO NO PLANO PTF (10 ${ }^{3}$ OTN) & 482,9 & 129,4 & 130,3 \\
PAR KM DE FIBRA & 1073,0 & 246,9 & 246,9 \\
TOTAL DE SISTEMAS NO PLANO PTF & 337 & 176 & 181 \\
NÚMERO DE UNIDADES MUX3 & 108 & 26 & 26 \\
NÚMERO DE UNIDADES MUX2 & 208 & 92 & 94 \\
TOTAL DE REPETIDORES OPTICOS & 83 & 19 & 19 \\
UTILIZAÇÃO DOS SIST. DE 34 Mbit/s & $39,0 \%$ & $84,6 \%$ & $87,0 \%$ \\
\hline
\end{tabular}

Tabela 2. Quadro resumo dos custos e necessidades de equipamento para a seqüência (P1, P2, P3).

5.2. Resultados para a Seqüência (P1, P3, P2, P3)

O carregamento dos arcos do plano PTF para esta seqüência é apresentado na Tabela 3, enquanto os resultados correspondentes a custos e equipamentos são dados na Tabela 4.

Observando as tabelas 2 e 4 pode-se notar que a segunda seqüência, embora roteando uma quantidade maior de sistemas PCM de $2 \mathrm{Mbit} / \mathrm{s}$ no plano PTF, conduz a uma solução de custo ligeiramente inferior àquela obtida a partir da primeira seqüência. Isto ocorre em todas as aplicações realizadas e se explica pelo fato de que na segunda seqüência a "aproximação" entre o custo real e o custo lenearizado se dá de forma menos abrupta. É claro que outras seqüências poderiam ser testadas para um melhor suporte à tomada de decisão.

Para a aplicação da segunda seqüência à rede de São Paulo, o tempo total de CPU foi de 75 minutos num computador PDP10.

Os limitantes inferiores para a solução ótima mencionados na Seção 3, quando identificados, correspondem ao valor do custo linearizado. Como não há garantia de que surja um bom limitante inferior numa determinada seqüência de passos, é, em princípio, recomendável que se procedam diversas experiências com diferentes seqüências de passos. 
FLUXO NOS ARCOS

\begin{tabular}{|c|c|c|c|c|c|c|}
\hline \multicolumn{2}{|c|}{ ARCOFIBRA } & \multirow{2}{*}{ 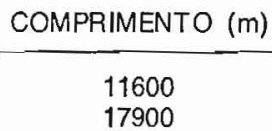 } & \multirow{2}{*}{$\begin{array}{r}\text { P1 } \\
14 \\
6\end{array}$} & \multirow{2}{*}{$\begin{array}{r}\text { P3 } \\
14 \\
0\end{array}$} & \multirow{2}{*}{$\begin{array}{r}\mathrm{P} 2 \\
0 \\
0\end{array}$} & \multirow{2}{*}{$\frac{P 3}{0}$} \\
\hline $\begin{array}{l}P D \\
P D\end{array}$ & $\begin{array}{ll}- & \mathrm{LI} \\
- & \mathrm{CB}\end{array}$ & & & & & \\
\hline PD & - $\quad A M$ & 22100 & 21 & 16 & 16 & 16 \\
\hline PD & - $\quad P E$ & 18400 & 10 & 10 & 10 & 10 \\
\hline PD & SM & 32300 & 1 & 0 & 0 & 0 \\
\hline PD & - SNE & 32100 & 10 & 10 & 10 & 10 \\
\hline $\mathrm{CO}$ & $-\quad \mathrm{CB}$ & 13700 & 6 & 0 & 0 & 0 \\
\hline $\mathrm{CO}$ & AM & 17900 & 9 & 9 & 0 & 0 \\
\hline $\mathrm{CO}$ & $\mathrm{PE}$ & 12700 & 2 & 0 & 0 & 0 \\
\hline $\mathrm{CO}$ & SM & 26600 & 1 & 0 & 0 & 0 \\
\hline $\mathrm{CO}$ & - SNE & 26800 & 4 & 0 & 0 & 0 \\
\hline PA & AM & 14500 & 5 & 0 & 0 & 0 \\
\hline PA & PE & 16100 & 3 & 0 & 0 & 0 \\
\hline PA & SM & 30000 & 2 & 0 & 0 & 0 \\
\hline PA & - SNE & 24500 & 12 & 16 & 16 & 16 \\
\hline VM & AM & 10800 & 12 & 0 & 0 & 0 \\
\hline VM & BR & 9500 & 4 & 0 & 0 & 0 \\
\hline VM & PE & 16100 & 2 & 0 & 0 & 0 \\
\hline VM & SM & 30700 & 1 & 0 & 0 & 0 \\
\hline VM & - SNE & 20800 & 4 & 0 & 0 & 0 \\
\hline $\mathrm{SI}$ & UI & 9900 & 2 & 0 & 0 & 0 \\
\hline SI & - $\quad P E$ & 14300 & 3 & 0 & 0 & 0 \\
\hline $\mathrm{SI}$ & - $\quad S M$ & 28200 & 1 & 0 & 0 & 0 \\
\hline si & - SNE & 29300 & 4 & 0 & 0 & 0 \\
\hline$B G$ & $-\quad C B$ & 4500 & 1 & 0 & 0 & 0 \\
\hline$B G$ & AM & 18700 & 28 & 28 & 28 & 28 \\
\hline$B G$ & PE & 12600 & 3 & 0 & 0 & 0 \\
\hline$B G$ & - SM & 26500 & 3 & 0 & 0 & 0 \\
\hline$B G$ & - SNE & 27600 & 8 & 12 & 12 & 12 \\
\hline AN & LI & 9700 & 1 & 0 & 0 & 0 \\
\hline AN & $\mathrm{CB}$ & 16400 & 2 & 0 & 0 & 0 \\
\hline AN & SM & 25800 & 2 & 0 & 0 & 0 \\
\hline LI & $C B$ & 11300 & 16 & 16 & 16 & 16 \\
\hline $\mathrm{LI}$ & AM & 15200 & 8 & 0 & 0 & 0 \\
\hline LI & PE & 12400 & 11 & 0 & 0 & 0 \\
\hline LI & SM & 26300 & 3 & 0 & 0 & 0 \\
\hline LI & - SIVE & 23400 & 11 & 15 & 15 & 15 \\
\hline $\mathrm{CB}$ & AM & 11500 & 49 & 48 & 48 & 48 \\
\hline $\mathrm{CB}$ & $B R$ & 16400 & 2 & 0 & 0 & 0 \\
\hline $\mathrm{CB}$ & PE & 23000 & 4 & 0 & 0 & 0 \\
\hline AM & $B R$ & 20300 & 9 & 9 & 0 & 0 \\
\hline$A M$ & $P E$ & 26900 & 7 & 7 & 0 & 0 \\
\hline AM & Su & 41500 & 1 & 0 & 0 & 0 \\
\hline AM & - SNE & 23200 & 11 & 10 & 9 & 10 \\
\hline BR & - $\quad 5 M$ & 21200 & 1 & 0 & 0 & 0 \\
\hline $\mathrm{BR}$ & - Sive & 22900 & 3 & 0 & 0 & 0 \\
\hline PE & - SM & 16200 & 10 & 12 & 12 & 12 \\
\hline $\mathrm{PE}$ & - Sive & 15600 & 3 & 0 & 0 & 0 \\
\hline SM & - SWE & 31800 & 1 & 0 & 0 & 0 \\
\hline \multicolumn{3}{|c|}{ TOTAL DO SISTEMA } & 337 & 232 & 197 & 193 \\
\hline
\end{tabular}

Tabela 3. Carregamento nos arcos do plano PTF para a seqüência (P1, P3, P2, P3). 


\begin{tabular}{l|c|c|c|c}
\multirow{2}{*}{} & \multicolumn{3}{|c}{ PASSOS } \\
\cline { 2 - 5 } & P1 & P3 & P2 & P3 \\
\hline CUSTO LINEARIZADO (10 3 OTN) & 746,1 & 798,2 & 801,6 & 795,6 \\
CUSTO REAL (103 OTN) & 1015,4 & 804,3 & 802,6 & 801,7 \\
CUSTO NO PLANO PTP (103 OTN) & 532,5 & 624,5 & 661,0 & 660,1 \\
CUSTO NO PLANO PTF (103 OTN) & 482,9 & 179,8 & 141,6 & 141,6 \\
PAR KM DE FIBRA & 1073,0 & 374,4 & 270,7 & 270,7 \\
TOTAL DE SISTEMAS NO PLANO PTF & 337 & 232 & 192 & 193 \\
NÚMERO DE UNIDADES MUX3 & 108 & 36 & 28 & 28 \\
NÚMERO DE UNIDADES MUX2 & 208 & 124 & 100 & 100 \\
TOTAL DE REPETIDORES OPTICOS & 83 & 27 & 21 & 21 \\
UTILIZAÇÃO DOS SIST. DE 34 Mbit/S & $39,0 \%$ & $80,6 \%$ & $85,7 \%$ & $86,2 \%$ \\
\hline
\end{tabular}

Tabela 4. Quadro resumo dos custos e necessidades de equipamento para a seqüência (P1, P3, P2, P3).

Vamos descrever algumas situações, primeiramente, através do segundo exemplo, onde foi utilizada a seqüencia ( $P 1, P 3, P 2, P 3)$. O resultado do passo $\mathrm{P} 1$ permite afirmar, com todo rigor matemático, que $\mathrm{V}_{1} 1=746,1 \mathrm{x}$ $10^{3}$ OTN é um limitante inferior para o problema, isto é, não é possivel encontrar solução ótima para o problema com custo inferior a $V 1$. O resultado do passo seguinte P3 não pode ser usado como limitante inferior por causa do valor obtido para o número de sistemas a rotear no arcofibra LI-SNE (note que neste caso o custo linearizado não é inferior ao custo real). $O$ resultado do último passo, também do tipo $P 3$, nos permite afirmar que; dentro de certas hipóteses restritivas, o valor $V 2=795,6 \times 10^{3}$ OTN constitui um limitante inferior para a solução ótima. Fora destas condições restritivas, este valor é apenas uma indicação. Tais condições restritivas referem-se ao número máximo de sistemas a rotear em cada arcofibra. Assim, para garantir que V2 é um limitante inferior temos que aceitar que o número máximo de sistemas a rotear é: (a) 4 para os arcofibras PD-LI, PD-CB, PD-PE, ..., SM-SNE, (b) 20 para os arcofibras PD-AM, PA-SNE e LI-CB, e outras condições deste tipo, uma para cada arcofibra. Se o tomador de decisão julgar aceitáveis as condições restritivas anteriormente descritas, poderá adotar a solução deste passo P3 sabendo que sua possível margem de melhoria não excede $(801,7-795,6) /(801,7) \cong 0,8 \%$.

Algumas vezes, a seqüência de passos é tal que inibe o aparecimento de "boas" soluções. Assim, no último passo do primeiro exemplo, onde foi utiliza- 
da a seqüência (P1, P2, P3) foi forçada uma situação, através das sucessivas linearizações, em que os arcofibras PD-SNE e PE-SM não são usados, o que gera uma solução relativamente pobre, com condições restritivas pouco aceitáveis. Fatos como este aconselham que um estudo só seja considerado completo quando diversas seqüências tiverem sido ensaiadas.

\section{Conclusões}

O sistema computacional desenvolvido para dimensionamento de fibras ópticas de $34 \mathrm{Mbit} / \mathrm{s}$, no planejamento da rede de troncos digitais em áreas locais multicentrais, foi utilizado inicialmente no planejamento da cidade de São Paulo, conforme exemplificado no presente trabalho. Posteriomente, foi também empregado nos estudos de planejamento das cidades do Rio de Janeiro e João Pessoa. Procurando tornar mais flexível o uso do programa, foi desenvolvida uma versão para microcomputadores compativeis com 0 IBM-PC. Encontra-se em desenvolvimento uma nova versão, onde são aperfeiçoados os algoritmos para cálculo de rotas e as heurísticas de construção dos custos. Tais mudanças são no sentido de reduzir os tempos computacionais obtidos. Nesta mesma versão também será incorporada a possibilidade de entroncamentos em $140 \mathrm{Mbit} / \mathrm{s}$ (1.920 canais).

A análise dos resultados obtidos com a apliçação do algoritmo proposto, descrita na Seção 5, comprova a "boa" qualidade da solução encontrada. O principal mérito do algoritmo é permitir o tratamento de um problema de natureza combinatória e com custos não-lineares e discretos, através de uma seqüência de problemas lineares por parte.

Uma continuidade natural do presente trabalho, a ser futuramente explorada, é o tratamento deste problema para um horizonte com multiperíodos.

\section{Referências}

[1] A. S. Garcia, "Planejamento do Entroncamento em Redes Telefônicas Urbanas em Processo de Digitalização", Tese de Doutorado, FEE/UNICAMP, 1987.

[2] Y. Rapp, "Planning of Junction Network in a Multi-Exchange Area: General Principles", Ericsson Technics, vol. 20, n. 1, 1964, pp. 77-130.

[3] Y. Rapp, "Planning of Junction Network in a Multi-Exchange Area: Extension of Principles and Applications", Ericsson Technics, vol.21, n. 2 , 1965, pp. 187-240.

[4] R. I. Wilkinson, "Theories for Toll Traffic Engineering in the USA", Bell System Technical Journal, vol. $35, n{ }^{\circ} 2,1956$, pp. 421-514. 
[5] B. Wallström, "Methods for Optimizing Alternative Routing Networks", Ericsson Technics, vol. 25, n. 1,1969, pp. 1-28.

[6] A. O. Moreno, "Planejamento de Redes Telefônicas: Determinação do Circuito de Junção Local", Tese de Mestrado, ITA, 1974.

[7] A. C. F. Reis, "Aplicações de Modelos de Programação Combinatória ao Problema de Expansão de Redes Telefônicas", Tese de Mestrado, COPPE/UFRJ, 1979.

[8] C. R. Tiba, L. R. Solon e J. M. Uehara, "Otimização do Entrocamento Local - OTELO", Relatório Interno, TELESP, 1981.

[9] M. Shibata, "Otimização do Investimento a Curto Prazo em Redes de Transmissão Telefônicas em Áreas Multicentrais", Tese de Mestrado, IMECC/UNICAMP, 1981.

[10] G. Robin e S. R. Treves, "Programatic Introduction of Digital Switching and Transmission in Existing Networks", IEEE Transactions on Communications, vol. COM-27, n. 7 , Julho 1979, pp. 1071-1078.

[11] G. J. McCallum, "A Generalized Upper Bounding Approach to a Communications Network Planning Problem", Networks, 1977, pp. 1-23.

[12] M. Minoux, J. Y. Serreault, "Synthèse Optimale d'un Réseaux de Télécommunications avec Constraints de Sécurité", Annales de Télécommunications, vol. 36, n. $3-4,1981$, pp. 211-230.

[13] A. S. Garcia, H. M. F. Tavares, R. V. Ribeiro e T. M. F. Bim, "Uma Metodologia para Otimização do Roteamento de Troncos em Redes Telefônicas Urbanas com Segurança", II Congresso Latino Americano de Investigation Operativa, 1984, Buenos Aires.

[14] B. Yaged, "Minimum Cost Routing for Dynamic Network Models", Networks, 3, 1973, pp. 193-224.

[15] A. S. Garcia, H. M. F. Tavares, R. V. Ribeiro e R. B. Guerra, "Um Procedimento para Otimização do Roteamento em Horizonte de Longo Prazo", Anais do VI Congresso Brasileiro de Automática, Belo Horizonte, 1986.

[16] I. Baybars e R. H. Edahl, "A Heuristic Method for Facility Planning in Telecommunications Networks with Multiple Alternate Routes", Naval Research Logistics, vol. 35, 1988, pp. 503-528. 
ANILTON SALLES GARCIA nasceu no Espírito Santo, em 1953. Concluiu o curso de Engenharia Mecânica na Universidade Federal do Espírito Santo (UFES), em 1976. Obteve o grau de Mestre em Matemática Aplicada (Otimização e Pesquisa Operacional) na UNICAMP, em 1978, e c grau de Doutor em Engenharia Elétrica na UNICAMP, em 1987. É Professor Adjunto do Centro Tecnológico da UFES, desde 1978, estando atualmente em regime de tempo parcial. Trabalha ainda no Departamento de Engenharia de Sistemas da ELEBRA TELECON LTDA, na área de Planejamento e Dimensionamento de Sistemas de Telecomunicações. Suas áreas de interesse atual são Estudos de Tráfego Telefônico, Dimensionamento de Sistemas de Telecomunicações, Planejamento de Redes Telefônicas, Digitalização de Redes e Programação Matemática.

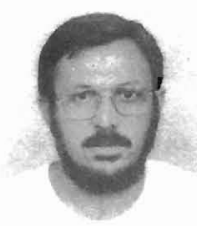

HERMANO DE M. F. TAVARES nasceu na Paraíba, em 1941. Concluiu o curso de Engenharia Eletrônica no Instituto Tecnológico da Aeronáutica (ITA) em 1964 e o Doutorado em Automática na Universidade de Toulouse em 1968. Trabalhou em várias áreas ligadas a Controle Automático e Otimização de Sistemas. Está atualmente no Departamento de Engenharia de Sistemas (DENSIS) da Faculdade de Engenharia Elétrica (FEE), UNICAMP, onde trabalha desde 1971. Suas áreas de interesse atual são Planejamento de Sistemas de Potência, Planejamento de Redes Telefônicas e Programação Matemática. 